\title{
Structural phase transitions on the nanoscale: The crucial pattern in the phase-change materials $\mathrm{Ge}_{2} \mathrm{Sb}_{2} \mathrm{Te}_{5}$ and $\mathrm{GeTe}$
}

\author{
J. Akola ${ }^{1,2}$ and R. O. Jones ${ }^{1}$ \\ ${ }^{1}$ Institut für Festkörperforschung, Forschungszentrum Jülich, D-52425 Jülich, Germany \\ ${ }^{2}$ Nanoscience Center, Department of Physics, P.O. Box 35, FI-40014 University of Jyväskylä, Finland \\ (Received 19 July 2007; revised manuscript received 27 September 2007; published 4 December 2007)
}

\begin{abstract}
Phase-change materials are of immense importance for optical recording and computer memory, but the structure of the amorphous phases and the nature of the phase transition in the nanoscale bits pose continuing challenges. Massively parallel density functional simulations have been used to characterize the amorphous structure of the prototype materials $\mathrm{Ge}_{2} \mathrm{Sb}_{2} \mathrm{Te}_{5}$ and $\mathrm{GeTe}$. In both, there is long-ranged order among Te atoms and the crucial structural motif is a four-membered ring with alternating atoms of types $A(\mathrm{Ge}$ and $\mathrm{Sb}$ ) and $B$ $(\mathrm{Te})$, an " $A B A B$ square." The rapid amorphous-to-crystalline phase change is a reorientation of disordered $A B A B$ squares to form an ordered lattice. There are deviations from the " $8-N$ rule" for coordination numbers, with Te having near threefold coordination. Ge atoms are predominantly fourfold coordinated, but — contrary to recent speculation-tetrahedral coordination is found in only approximately one-third of the Ge atoms. The average coordination number of $\mathrm{Sb}$ atoms is 3.7, and the local environment of $\mathrm{Ge}$ and $\mathrm{Sb}$ is usually "distorted octahedral" with $A B$ separations from 3.2 to $4 \AA$ in the first coordination shell. The number of $A-A$ bonds is significantly greater in $\mathrm{GeTe}$ than in $\mathrm{Ge}_{2} \mathrm{Sb}_{2} \mathrm{Te}_{5}$. Vacancies (voids) in the disordered phases of these materials provide the necessary space for the phase transitions to take place. The vacancy concentration in $\mathrm{Ge}_{2} \mathrm{Sb}_{2} \mathrm{Te}_{5}$ $(11.8 \%)$ is greater than in GeTe $(6.4 \%)$, which is consistent with the better phase-change performance of the former.
\end{abstract}

DOI: 10.1103/PhysRevB.76.235201

PACS number(s): 61.43.Dq, 61.43.Bn, 61.46.-w, 71.15.Pd

\section{INTRODUCTION}

Modern computers and other electronic devices place ever-increasing demands on the density, speed, and stability of memory. Phase-change (PC) materials already play important roles in rewritable media [CD-RW, DVD-RW, DVDRAM, and Blu-ray Disc (BD)] and are prime candidates for wider applications in the future. ${ }^{1-4}$ The basis of their function is the rapid and reversible transition between the crystalline and amorphous forms in nanoscale bits $(\sim 100 \mathrm{~nm})$ of an alloy. ${ }^{5}$ The latter arises from quenching after a localized and short $(\sim 1 \mathrm{~ns})$ laser annealing to a temperature above the melting point $T_{m}$. Longer laser heating $(\sim 50 \mathrm{~ns})$ to above the glass transition temperature but below $T_{m}$ leads to a metastable crystalline form. The changes in the optical and electronic properties that accompany the transition provide ready means to monitor it.

The most common PC materials are tellurium-based alloys, with the $\mathrm{Ge}_{x} \mathrm{Sb}_{y} \mathrm{Te}_{1-x-y}$ family providing prototypes. $\mathrm{Ge}_{x} \mathrm{Te}_{1-x}$ alloys were the first to show real promise as PC storage media, ${ }^{6}$ and the widely studied $\mathrm{Ge}_{2} \mathrm{Sb}_{2} \mathrm{Te}_{5}$ (GST) is used commercially in DVD-RAM (random access memory). Central to our understanding of the properties of these materials is a knowledge of the structures of the different phases, but these are difficult to determine in binary or ternary alloys with significant numbers of vacancies. As noted recently, "the local structural order of glasses in the ternary system $\mathrm{Ge}_{x} \mathrm{Sb}_{y} \mathrm{Te}_{1-x-y}$ is not well established." Even the structure of the ordered phase of GST is controversial: Yamada ${ }^{8}$ proposed that the metastable phase has a rocksalt structure with "Na" sites occupied randomly by $\mathrm{Ge}$ and $\mathrm{Sb}$ atoms and vacancies, and "Cl" sites by Te. Kolobov et al., ${ }^{9}$ however, proposed that $\mathrm{Ge}$ and $\mathrm{Sb}$ atoms are displaced from their ideal positions, enabling the order-disorder transition to occur as an "umbrella flip" of Ge atoms from octahedral to tetrahedral positions. A model of amorphous $\mathrm{Ge}_{1} \mathrm{Sb}_{2} \mathrm{Te}_{4}$ where a spinel structure (tetrahedral $\mathrm{Ge}$ ) was compared to the octahedral rocksalt phase has also been studied. ${ }^{10}$ Most recently, x-ray fluorescence holography of an epitaxial layer of GST indicated a cubic structure with tetrahedral site symmetry about Ge atoms. ${ }^{11}$ It is remarkable that phase-change materials could become the basis of commercially successful products with so much uncertainty about the structures of the phases involved.

Recent studies have begun to change this situation. Extended $\mathrm{x}$-ray absorption fine structure (EXAFS) measurements on GST have found significant concentrations of $\mathrm{Ge}-\mathrm{Ge}$ bonds and indications that overcoordinated Te atoms play a role, ${ }^{12}$ and there has been renewed focus on the role of vacancies. ${ }^{13,14}$ Density functional (DF) calculations suggest that the metastable structure is consistent with rocksalt symmetry but comprises two units ordered along the [111] direction. Higher temperatures lead to two-dimensional linear or tangled clusters that are ordered in the perpendicular direction. ${ }^{15} \mathrm{DF}$ simulations of liquid $\mathrm{Ge}_{1} \mathrm{Sb}_{2} \mathrm{Te}_{4}$ at $973 \mathrm{~K}$ indicated that all atoms have octahedral coordination. ${ }^{16}$ The reverse Monte Carlo (RMC) analysis of synchrotron radiation data from amorphous $(a-)$ GST and $a$-GeTe suggests that the ring structure of the former is dominated by fourand six-membered rings also seen in the crystal. ${ }^{17}$ The RMC method optimizes the form of various partial pair distribution functions from the measured structure factor $S(Q)$, but the results are sensitive to the constraints assumed, such as the presence or absence of homopolar bonds. Ring distributions are the basis of a rate equation model of the amorphous-tocrystalline phase transition in network materials ${ }^{18}$ in a two- 
dimensional network of fourfold coordinated atoms, the crystalline phase is represented by a square lattice and an amorphous bit by a region where several ring sizes coexist.

Density functional calculations are free of adjustable parameters, and their combination with molecular dynamics has had a major impact on materials science and chemistry. They have been used widely in chalcogenide materials. ${ }^{19}$ Nevertheless, their demands on computational resources have restricted simulations on GST systems to relatively small unit cells (up to 56 atoms in Ref. 10, 108 atoms and 12 vacancies in Ref. 15, and 168 atoms in Ref. 16) and time scales that are often much shorter than those measured (e.g., a total of 18 ps in Ref. 16). Here, we present the results of DF calculations of GST and GeTe that are much larger in both number of atoms (460 for GST and 216 for GeTe) and time scale (hundreds of picoseconds). The simulations are then close to the experimental time scale of $1 \mathrm{~ns}$ for the quenching process. Our simulations start from liquids at $3000 \mathrm{~K}$ and do not favor particular crystal types. We provide details of the geometries and electronic structures of the amorphous materials, and compare with experiment where possible.

\section{NUMERICAL METHODS}

\section{A. Density functional calculations}

The density functional calculations were performed with the Car-Parrinello molecular dynamics package ${ }^{20}$ (CPMD) using the generalized gradient approximation of Perdew, Burke, and Ernzerhof ${ }^{21}$ (PBE) for the exchange-correlation energy functional $E_{x c}$. For a range of systems (including chalcogen clusters), this approximation leads to improvements over the results of local density calculations for structures and energy differences. The electron-ion interaction is described by ionic pseudopotentials with the nonlocal, normconserving, and separable form of Troullier and Martins. ${ }^{22}$ The valence configurations are $4 s^{2} 4 p^{2}, 5 s^{2} 5 p^{3}$, and $5 s^{2} 5 p^{4}$ for $\mathrm{Ge}, \mathrm{Sb}$, and $\mathrm{Te}$, respectively, and the scalar-relativistic pseudopotentials contain nonlinear core corrections. ${ }^{23}$ The program employs periodic boundary conditions, usually with one point $(\mathbf{k}=0)$ in the Brillouin zone, and the kinetic energy cutoff of the plane wave basis set is $20 \mathrm{Ry}$.

GST is modeled at the densities of the metastable crystalline $\left(6.35 \mathrm{~g} / \mathrm{cm}^{3}\right)$ and amorphous $\left(5.88 \mathrm{~g} / \mathrm{cm}^{3}\right)$ phases. The simulations are based on the rocksalt lattice (crystalline GST) with 512 atomic sites, where the $\mathrm{Na}$ and $\mathrm{Cl}$ sites are occupied by $\mathrm{Ge}$ and/or $\mathrm{Sb}$ atoms (20\% each) and vacancies (10\%), and Te atoms $(50 \%)$, respectively. The $\mathrm{Na}$ sites are populated randomly with $\mathrm{Ge}, \mathrm{Sb}$, and vacancies, so that the system contains 460 atoms (102 Ge, $102 \mathrm{Sb}$, and $256 \mathrm{Te})$ and 52 vacancies. The size of the cubic simulation boxes for the crystalline and amorphous phases are 24.05 and $24.62 \AA$, respectively. The GeTe system contains 216 atoms at the density of the amorphous phase $\left(5.61 \mathrm{~g} / \mathrm{cm}^{3}\right)$, and the box size is $18.61 \AA$. Ge, $\mathrm{Sb}$, and Te are relatively heavy atoms, and Born-Oppenheimer molecular dynamics (MD) (optimization of electron density for each ionic configuration) is effective for simulations at high temperatures, particularly if - as in the present cases- the material is metallic. In addi- tion, the time steps adopted [250 and 125 a.u. (6.050 and $3.025 \mathrm{fs}$ ) for initialization and data collection, respectively] are much longer than in the Car-Parrinello approach.

The minimization of the energy functional during the simulations uses the direct inversion of the iterative subspace (DIIS). Preconditioning techniques, such as DIIS, are often used to find iterative solutions of large systems of linear equations. ${ }^{24}$ It has proven to be a rapidly convergent and robust method under a great range of circumstances, including the small or vanishing gaps between valence and condution bands found in GST and GeTe at high temperatures. The dynamics are performed using the temperature-independent PBE functional, but we note that DF calculations provided a very good description of the thermal expansion of the glass ceramic $\beta$-eucryptite $\left(\beta\right.$ - $\left.\mathrm{LiAlSiO}_{4}\right)$ over a temperature range of $1000 \mathrm{~K}$ (Ref. 25) without requiring a $T$-dependent form of $E_{x c}$. A detailed analysis of the electronic structure for selected geometries (snapshots) has been carried out with the Lanczos diagonalization scheme and the free energy functional of Alavi et al. $(T=1000 \mathrm{~K}){ }^{26}$

Memory of the crystalline starting structure was erased by starting the simulations at $3000 \mathrm{~K}$ (liquid), followed by gradual cooling over $42 \mathrm{ps}$ to the melting point $(900 \mathrm{~K})$. The first data collection was performed for $21 \mathrm{ps}$ at $900 \mathrm{~K}$, followed by cooling to $300 \mathrm{~K}$ over $139 \mathrm{ps}$. The second data collection (at $300 \mathrm{~K}$ ) was also performed for 21 ps. Finally, the system was quenched to $100 \mathrm{~K}$ over $74 \mathrm{ps}$, and the resulting structure was optimized using Car-Parrinello MD and simulated annealing. A similar cooling procedure for $\mathrm{GeTe}$ took a total of $340 \mathrm{ps}$, including data collection at $1000 \mathrm{~K}$ (45 ps) and $300 \mathrm{~K}$ (26 ps). Such relaxation times are essential, particularly for structures that are undergoing an ordering transition. Shorter time scales lead, for example, to overestimates of the number of Te-Te bonds and underestimates of the degree of order.

\section{B. Data analysis}

MD methods allow us to follow the coordinates $R_{i}$ and velocities $v_{i}$ of all atoms, and insight into the local order can be found from the distributions of the bond $\left(\theta_{i j k}\right)$ and dihedral angles $\left(\gamma_{i j k l}\right)$. The radial distribution function (RDF) or pair correlation function $g(r)$ is a spherically averaged distribution of interatomic vectors,

$$
g(r)=\frac{1}{\rho^{2}}\left\langle\sum_{i} \sum_{i \neq j} \delta\left(r_{i}\right) \delta\left(r_{j}-r\right)\right\rangle,
$$

where $\rho$ is the density. Partial radial distribution functions $g_{\alpha \beta}(r)$ can be calculated by restricting the analysis to the elements $\alpha$ and $\beta$. The local structure can also be characterized by the average coordination numbers, which are found by integrating $g_{\alpha \beta}(r)$ to the first minima $R_{\min }$

$$
n_{\alpha \beta}=\int_{0}^{R_{\min }} d r 4 \pi r^{2} \rho_{\alpha \beta}(r) g_{\alpha \beta}(r) .
$$

We calculate the structure factor $S(Q)$ by Fourier transforming the $g_{\alpha \beta}(r)$ to give the partial structure factors $S_{\alpha \beta}(Q)$ : 


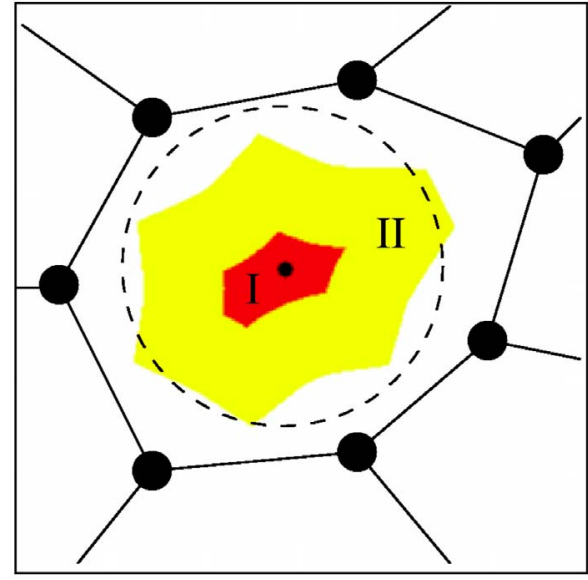

FIG. 1. (Color online) Schematic form for 2D vacancy.

$$
S_{\alpha \beta}(Q)=\delta_{\alpha \beta}+\rho_{\alpha \beta} \int_{0}^{\infty} d r 4 \pi r^{2}\left[g_{\alpha \beta}(r)-1\right] \frac{\sin (Q r)}{Q r} .
$$

These quantities are then weighted according to the atomic fractions $c_{\alpha}$ and form factors $f_{\alpha}(Q)$ (for $\mathrm{x}$ rays $x$ ) or the $Q$-independent coherent scattering lengths $b_{\alpha}$ (for neutrons $n),{ }^{27}$

$$
S^{n, x}(Q)=\sum_{\alpha, \beta \geqslant \alpha} w_{\alpha \beta}(Q) S_{\alpha \beta}(Q),
$$

where

$$
w_{\alpha \beta}=\frac{c_{\alpha} c_{\beta} f_{\alpha}(Q) f_{\beta}(Q)}{\left[\sum_{\alpha} c_{\alpha} f_{\alpha}(Q)\right]^{2}}\left(2-\delta_{\alpha \beta}\right)
$$

and

$$
\sum_{\alpha, \beta \geqslant \alpha} w_{\alpha \beta}(Q)=1 \quad \text { for all } Q .
$$

If the ratio of the form factors of the elements is weakly $Q$-dependent, we can replace $f(Q)$ by the atomic numbers $Z$ (Ge: 32, Sb: 51, and Te: 52).

In discussing the topology of nearest neighbors, it is convenient to separate the atoms into types $A(\mathrm{Ge}$ and $\mathrm{Sb}$ ) and $B$ (Te). To quantify the local order, we use order parameters $\alpha_{x}$ $\left(x=\mathrm{Ge}, \mathrm{Sb}\right.$, and Te) based on the theory of binary liquids: $:^{28}$

$$
\alpha_{x}=\frac{1-n_{A B} /\left[c_{A} c_{B}\left(n_{A}+n_{B}\right)\right]}{1-n_{x} /\left[c_{A} c_{B}\left(n_{A}+n_{B}\right)\right]},
$$

where $c_{A}$ and $c_{B}$ are the concentrations of the atom types $A$ and $B, n_{A}$ and $n_{B}$ are the total coordination numbers, and $n_{A B}$ is the corresponding $A B$ coordination number. Perfect order ( $A B$ alternation) corresponds to $\alpha_{x}=1$, a random mixture to $\alpha_{x}=0$, and complete phase separation to $\alpha_{x}=-1$.

The cavity analysis is aided by introducing several concepts illustrated in Fig. 1 for a two-dimensional system. A vacancy domain (I, red) is a region where the minimum distance to a nearby atom is larger than a certain cutoff (here, $85 \%$ of the bond length, which in the simulations is $2.8 \AA$, the average Ge-Te distance), and each domain is characterized by the point where the distance to all atoms is a maximum. If there are no maxima closer than the divacancy cutoff (2.4 $\AA$ in the simulations), we insert a test particle at the center of the largest sphere that can be placed inside the cavity (dashed circle in Fig. 1). This can be used to calculate RDF, including vacancy-vacancy correlations. A vacancy cell (II, yellow in Fig. 1) is analogous to the Wigner-Seitz cell in crystals or Voronoi polyhedra in amorphous materials, and refers to a whole cavity, i.e., a divacancy forms a single cavity. The assignment of such volumes was made using a mesh of $0.087 \AA$. The vacancy analysis is an additional reason for choosing large simulation cells. There are far fewer test particles than atoms, and the statistics are more sensitive to periodic boundary conditions in smaller simulations because vacancies are usually much larger than the atomic cells.

The eigenvalues of the density functional equations cannot be related directly to excitations in the system, but they provide information about the nature of the orbitals at different energies and gaps in the energy spectrum. The electronic density of states (DOS) can be written as

$$
N(E)=\sum_{\mathbf{k}} E_{\mathbf{k}} w_{\mathbf{k}} g\left(E-E_{\mathbf{k}}\right)
$$

where $E_{\mathbf{k}}$ are the eigenvalues of a $\mathbf{k}$ point in the (supercell) Brillouin zone, and $w_{\mathbf{k}}$ the corresponding weight. The Gaussian function has a width of $0.02 \mathrm{eV}$. In addition to calculations performed with $\mathbf{k}=0$, we use a $2 \times 2 \times 2$ mesh with at least four distinct $\mathbf{k}$ points. The DOS can be projected onto individual atom types and atomic functions.

Dynamical information includes the velocity autocorrelation function $C_{v}$ :

$$
C_{v}(t)=\frac{1}{N} \sum_{i=1}^{N} \frac{\left\langle v_{i}(0) \cdot v_{i}(t)\right\rangle}{\left\langle v_{i}(0) \cdot v_{i}(0)\right\rangle},
$$

where $N$ is the number of particles. The self-diffusion constant for all atoms or those of species $\alpha$ can be determined from $C_{v}$ :

$$
D_{\alpha}=\frac{1}{3} \int_{0}^{\infty} d t C_{v}(t),
$$

or directly from the coordinates $R_{\alpha}$ :

$$
D_{\alpha}=\lim _{t \rightarrow \infty} \frac{\left\langle\left|R_{\alpha}(t)-R_{\alpha}(0)\right|^{2}\right\rangle}{6 t},
$$

where the average is over all atoms of species $\alpha$. The connection between the self-diffusion constant $D$ and the viscosity $\eta$ is well known, and the Stokes-Einstein relation ( $D \eta a / k_{B} T=$ const) holds in many liquids. Here, $a$ is the particle diameter, and the constant depends on the choice of boundary conditions. Highly supercooled liquids and other systems can show large deviations from this behavior. ${ }^{29}$ 


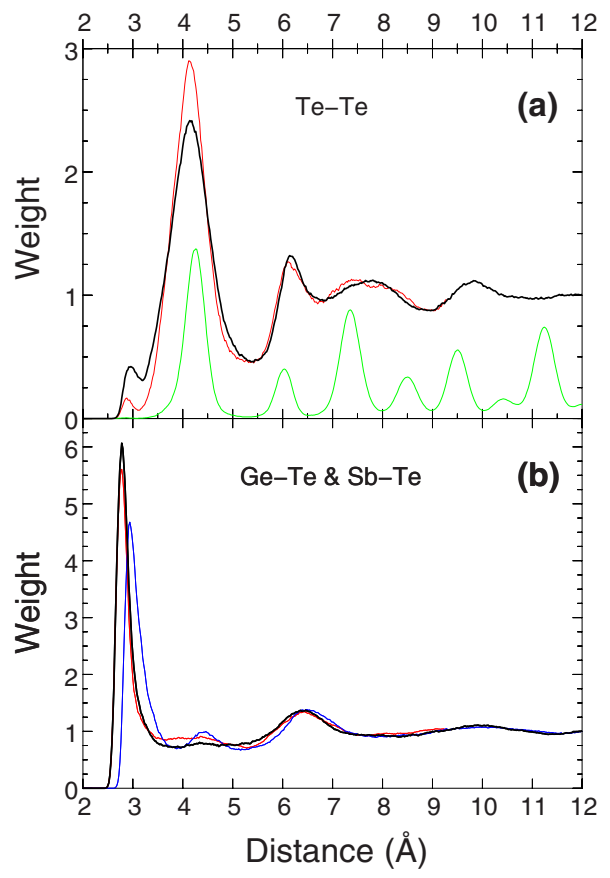

FIG. 2. (Color online) Radial distribution functions of $a$-GST and $a$-GeTe at $300 \mathrm{~K}$. (a) Te-Te [black, $a$-GST; red, $a$-GeTe; and green (scaled by 0.25), metastable GST crystal at $300 \mathrm{~K}$ ]. (b) Partial RDF (black, Ge-Te bonds in $a$-GST; red, Ge-Te bonds in $a$-GeTe; and blue, $\mathrm{Sb}$-Te bonds in $a$-GST).

\section{RESULTS}

\section{A. Radial distribution functions and structure factors}

The partial RDF are shown in Fig. 2. It is clear from the Te-Te curve [Fig. 2(a)] that amorphous GeTe and GST have striking long-range correlations of Te atoms up to $10 \AA$, which is another indication that large simulation cells are necessary. There are few Te-Te bonds $(0.1$ and 0.3 per Te atom for $a$-GeTe and $a$-GST) and the second maximum at $4.16 \AA$ dominates. The region below $7 \AA$ shows parallels to the ordered structure (rocksalt). There are differences at larger distances, and the cubic Te sublattice proposed in the umbrella flip model ${ }^{9}$ is not found. The corresponding homopolar RDF for the remaining atoms (Ge and Sb: type $A$ ) exhibit little structure beyond $\sim 6 \AA$ and are not shown.

GeTe and GST form mainly Ge-Te (and Sb-Te) bonds with pronounced first maxima in the partial RDF [Fig. 2(b) and Table I]. The Ge-Te and Sb-Te bonds are shorter than in the crystal, but the corresponding maxima at 2.78 and $2.93 \AA$ are larger than those found in the EXAFS measurements. ${ }^{9,12}$ Nevertheless, the ranges of the calculated and experimental bond lengths overlap. The location of the first minimum usually marks the limit of bond lengths, but the minima in $a$-GeTe and $a$-GST move to $3.6-3.9 \AA$, so that there are many Ge-Te (and Sb-Te) pairs with "intermediate" separations. The most prominent topologies are $\mathrm{Ge}_{-} \mathrm{Te}_{4}, \mathrm{Sb}-\mathrm{Te}_{3}$, and $\mathrm{Te}-\mathrm{A}_{3}$ for $a$-GST, and $\mathrm{Ge}-\mathrm{GeTe}_{3}$ and $\mathrm{Te}-\mathrm{Ge}_{3}$ for $a$-GeTe. Te chains, often postulated in Te-based systems, are not evident, and the average coordination number of $\mathrm{Te}$ is near 3 in amorphous GeTe and GST.

Amorphous GST has $\mathrm{Ge}-\mathrm{Ge}, \mathrm{Sb}-\mathrm{Sb}$, and $\mathrm{Ge}-\mathrm{Sb}$ bonds with coordination numbers [Eq. (2)] 0.4, 0.6, and 0.2, respectively. Baker et al. ${ }^{12}$ have reported an EXAFS value of $0.6 \pm 0.2$ for $\mathrm{Ge}-\mathrm{Ge}$ bonds, although other homopolar bonds could not be assigned. Ge-Ge bonds are more common in $a$-GeTe, where our coordination number (1.1) compares well with an EXAFS measurement (1.2). ${ }^{30}$ All total coordination numbers (Table I) are larger than given by the " $8-N$ rule" (Ge: 4, Sb: 3, and Te: 2), and the coordination of Te atoms is

TABLE I. Calculated structural properties of $\mathrm{Ge}_{2} \mathrm{Sb}_{2} \mathrm{Te}_{5}$ and $\mathrm{GeTe}$ at $300 \mathrm{~K}: \mathrm{Ge}-\mathrm{Te}$ and $\mathrm{Sb}-\mathrm{Te}$ bond lengths $\left(r_{0}\right)$ and the first minima $\left(r_{\text {min }}\right)$ of the partial RDF, atomic coordination numbers $\left(n_{x}\right.$, cutoff distance $3.2 \AA)$ and order parameters $\left(\alpha_{x}\right)$, and the total volume of cavities $\left(V_{v}\right)$.

\begin{tabular}{|c|c|c|c|c|c|c|c|c|}
\hline & \multicolumn{2}{|c|}{$a-\mathrm{Ge}_{2} \mathrm{Sb}_{2} \mathrm{Te}_{5}$} & \multicolumn{2}{|c|}{$c-\mathrm{Ge}_{2} \mathrm{Sb}_{2} \mathrm{Te}_{5}$} & \multicolumn{2}{|c|}{$a-\mathrm{GeTe}$} & \multicolumn{2}{|c|}{$c-\mathrm{GeTe}$} \\
\hline & Calculated & Expt. & Calculated & Expt. & Calculated & Expt. & Calculated & Expt. \\
\hline \multirow[t]{2}{*}{$r_{0}(\AA)$} & \multirow[t]{2}{*}{$2.78 / 2.93$} & $2.61 / 2.85^{\mathrm{a}}$ & \multirow[t]{2}{*}{$2.92 / 3.02$} & $2.83 / 2.91^{\mathrm{a}}$ & \multirow[t]{2}{*}{2.78} & \multirow[t]{2}{*}{$2.65^{\mathrm{b}}$} & \multirow[t]{2}{*}{3.00} & $3.00^{\mathrm{b}}$ \\
\hline & & $2.63 / 2.83^{c}$ & & $3.0 \pm 0.3^{\mathrm{a}}$ & & & & $2.80,2.84^{\mathrm{b}}$ \\
\hline$r_{\min }(\AA)$ & \multicolumn{4}{|l|}{$3.84 / 3.88$} & \multicolumn{2}{|l|}{3.68} & \\
\hline$n_{\mathrm{Ge}}$ & 4.2 & $3.9 \pm 0.8^{\mathrm{c}}$ & \multicolumn{2}{|c|}{6} & 4.2 & $3.7^{\mathrm{b}}$ & \multicolumn{2}{|c|}{$3+3$} \\
\hline$n_{\mathrm{Sb}}$ & 3.7 & $2.8 \pm 0.5^{\mathrm{c}}$ & \multicolumn{2}{|c|}{6} & & & & \\
\hline$n_{\mathrm{Te}}$ & 2.9 & $2.4 \pm 0.8^{\mathrm{c}}$ & \multicolumn{2}{|c|}{$4.8^{\mathrm{d}}$} & 3.3 & $2.5^{\mathrm{b}}$ & \multicolumn{2}{|c|}{$3+3$} \\
\hline$\alpha_{\mathrm{Ge}}$ & 0.77 & & \multicolumn{2}{|c|}{1} & 0.56 & & \multicolumn{2}{|c|}{1} \\
\hline$\alpha_{\mathrm{Sb}}$ & 0.61 & & \multicolumn{2}{|c|}{1} & & & & \\
\hline$\alpha_{\mathrm{Te}}$ & 0.75 & & \multicolumn{2}{|c|}{1} & 0.94 & & \multicolumn{2}{|c|}{1} \\
\hline$V_{v}(\%)$ & 11.8 & & \multicolumn{2}{|c|}{$\sim 10$} & 6.4 & & \multicolumn{2}{|c|}{$\sim 0$} \\
\hline
\end{tabular}

${ }^{\mathrm{a}}$ EXAFS and XRD (Ref. 9).

${ }^{b}$ EXAFS and XRD (Ref. 30), and ND (Ref. 31). $c$-GeTe structures: rhombohedral (shorter bonds, coordination $3+3$ ) and fcc (longer bonds, coordination 6).

${ }^{\mathrm{c}}$ EXAFS (Ref. 12).

${ }^{\mathrm{d}}$ Vacancies reduce the value below 6 . 


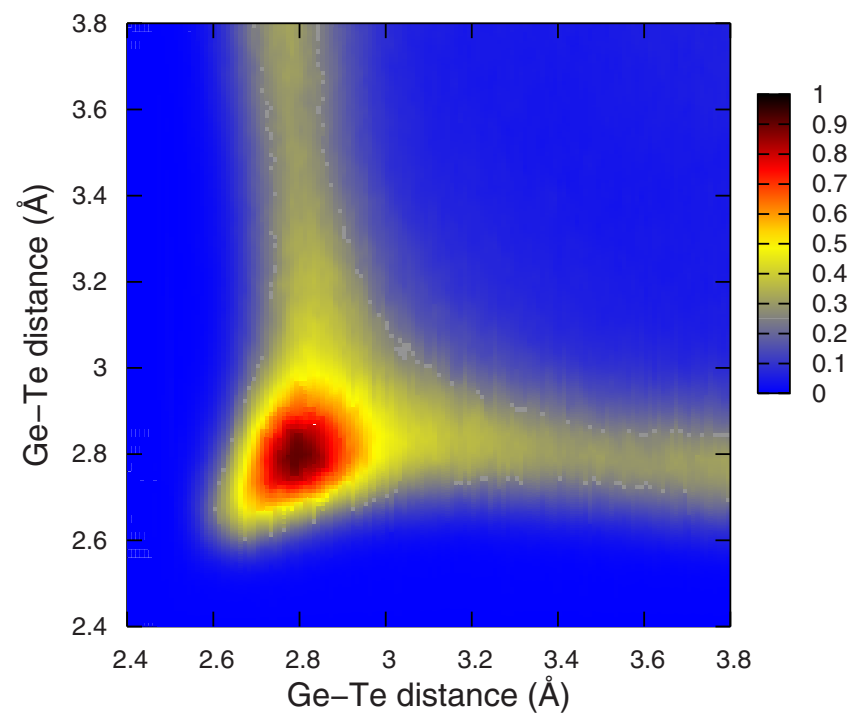

FIG. 3. (Color online) Distance-distance correlation map of Ge-Te bonds in $a$-GST at $300 \mathrm{~K}$ (with respect to Ge atoms).

lower in $a$-GST. The overall coordination numbers are 3.4 in $a$-GST and 3.7 in $a$-GeTe.

Further insight into the structure near a Ge atom in $a$-GST can be obtained from Fig. 3, which shows the relative probability of occurrence of pairs of Ge-Te bonds of particular lengths (the distance-distance correlation map). The intermediate distances are seen here as bridges that correlate mainly with Ge-Te bonds of length $2.8 \AA$. The "arrowhead" profile of the map indicates that some Ge atoms prefer shorter bonds to Te with less correlation with intermediate separations (3.2-4.0 A). The calculated order parameters [Eq. (7) and Table I] show that the amorphous materials are "semiordered" (values above 0.5), i.e., the structure shows a clear alternation of atomic types but is not crystalline $(\alpha=1)$, and the coordination is lower. Ge is more disordered than Te in $a$-GeTe (Ge-Ge bonds), whereas $\mathrm{Sb}$ is the least ordered in $a$-GST.

The structure factors $S(Q)$ of $a$-GST have been calculated at $300 \mathrm{~K}$ (Fig. 4) and $900 \mathrm{~K}$ (liquid, Fig. 5). There are only minor differences between the $S(Q)$ calculated for neutrons and $\mathrm{x}$ rays, and the curves calculated from the total $g(r)$ (where the elements have equal weight) are also very similar. While the calculated peak positions tend to be at slightly lower $Q$ values than those measured, all features are resolved. The structure factor for the amorphous phase has more structure and sharper peaks than for the liquid phase. There are more homopolar bonds (less $A B$ alternation) and more disorder in the latter.

\section{B. Angular distribution functions}

Apart from $\mathrm{Ge}-\mathrm{Te}$ and $\mathrm{Sb}$-Te, all partial RDFs of $a$-GST and $a$-GeTe have minima near 3.1-3.2 $\AA$, and a cutoff distance of $3.2 \AA$ has been used for the angular distribution of $\mathrm{Te}-\mathrm{Ge}-\mathrm{Te}$ and Te-Sb-Te configurations (Fig. 6, where configurations are included if both bonds are shorter than $3.2 \AA$ ) and for the analysis of irreducible rings (see below, Fig. 7).

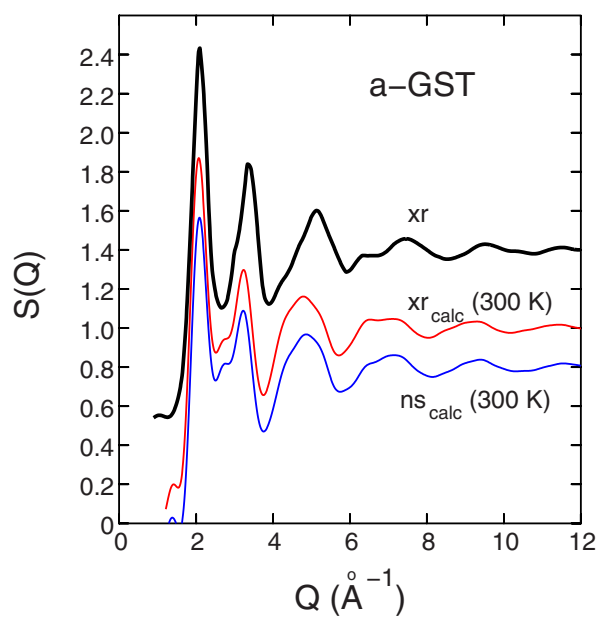

FIG. 4. (Color online) Structure factor $S(Q)$ of $a$-GST. The curve of Kohara et al. (xr, Ref. 17) is displaced by 0.4.

The angular distributions (Fig. 6) show octahedral features (a strong maximum at $90^{\circ}$ and a weaker peak at $180^{\circ}$ ), and $\mathrm{Sb}$ in $a$-GST is more strongly peaked than Ge. There is little weight below $60^{\circ}$ and above $120^{\circ}$. The broad peak at $90^{\circ}$ includes tetrahedral bond angles $\left(109.47^{\circ}\right)$, and the distribution of Ge-Te bonds shorter than $2.7 \AA$ is peaked closer to this value. While $60 \%$ of the Ge atoms are fourfold coordinated in both $a$-GeTe and $a$-GST, far fewer (34\% in $a$-GST and $29 \%$ in $a$-GeTe) are tetrahedrally bonded. ${ }^{33}$ In contrast to the conclusion of Kolobov et al. based on x-ray absorption near edge structure measurements, ${ }^{9}$ tetrahedral Ge atoms are not the main structural motif of the amorphous materials. Sb does not favor tetrahedral coordination under any circumstances.

The dihedral angles around $\mathrm{Ge}-\mathrm{Te}$ (and $\mathrm{Sb}-\mathrm{Te}$ ) bonds (Fig. 6, inset) show pronounced maxima at $0^{\circ}, 90^{\circ}$, and $180^{\circ}$ that are consistent with octahedral (cubic) coordination. This is further evidence of the similarities between the amorphous and crystalline phases.

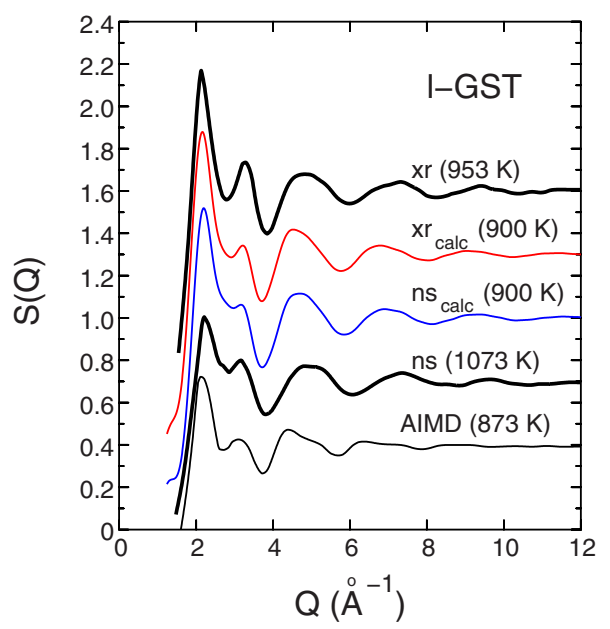

FIG. 5. (Color online) Structure factor $S(Q)$ for the liquid state of GST. "xr," Kohara et al. (Ref. 17); "ns" and "AIMD," neutron scattering and AIMD results of Ref. 32. Successive curves are displaced by 0.3 . 


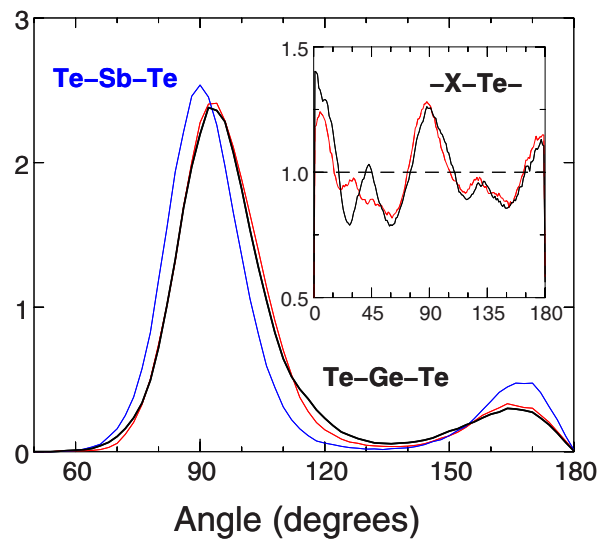

FIG. 6. (Color online) Angular distributions at $300 \mathrm{~K}$. Black, Te-Ge-Te in $a$-GST; red, Te-Ge-Te in $a$-GeTe; and blue, Te-Sb-Te in $a$-GST. Inset: Dihedral angles around $-\mathrm{X}-\mathrm{Te}-$ bonds $(X=\mathrm{Ge}$ or $\mathrm{Sb})$. Black, $a$-GST; red, $a$-GeTe.

\section{Ring structures}

Irreducible rings (the shortest closed loops) are important in the theory of amorphous materials, as they can be used to express the entropy of disorder ${ }^{34,35}$ and to characterize the phase transition between the crystalline and amorphous states. ${ }^{18}$ The statistics for different ring sizes (Fig. 7) show clear differences between amorphous GST and GeTe: $a$-GST has a pronounced maximum at four-membered rings, the weight of larger rings decreases, and there is a pronounced odd-even alternation. It is striking that $86 \%$ of the fourmembered rings have $A B A B$ alternation, and this is also observed for $a$-GeTe. Since the bond angle distributions are peaked around $90^{\circ}$, we refer to these structural units as " $A B A B$ squares." We find that $60 \%-80 \%$ of Ge atoms par-

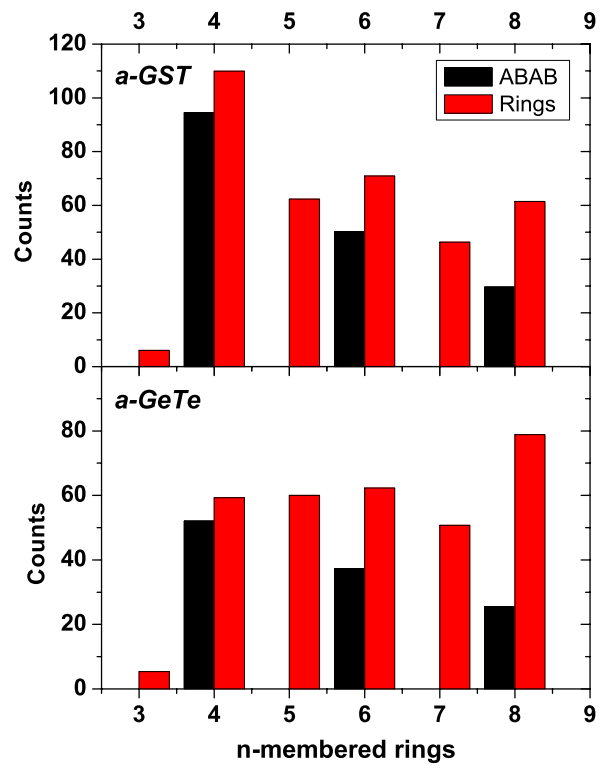

FIG. 7. (Color online) Statistics of irreducible $n$-fold ring configurations of $a$-GST and $a$-GeTe at $300 \mathrm{~K}$. Black, corresponding alternating $A B$ configurations ( $A, \mathrm{Ge}$ and/or $\mathrm{Sb} ; B, \mathrm{Te}$ ).

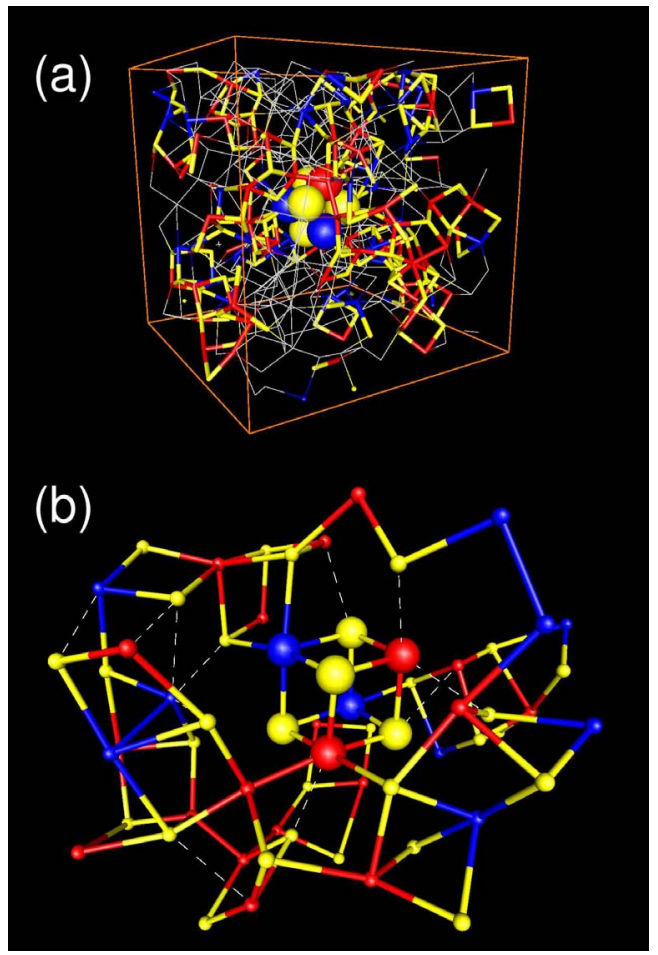

FIG. 8. (Color online) $A B A B$ squares and cubes in $a$-GST. (a) Simulation box of $a$-GST (24.6 $\AA$, 460 atoms) with atoms and bonds of $A B A B$ squares and cube highlighted. Red, Ge; blue, Sb; and yellow, Te. (b) Local environment of $A B A B$ cube. Dashed lines mark intermediate distances $(3.2-4.0 \AA)$.

ticipate in at least one $A B A B$ square depending on the bond cutoff distance chosen $(3.2-3.4 \AA)$. The RMC analysis of x-ray diffraction measurements of $S(Q)$ led to ring statistics, ${ }^{17}$ but these authors did not discuss alternating $A B$ configurations.

The large number of alternating four-membered rings is evident in Fig. 8(a), where we have highlighted the relevant atoms and bonds in $a$-GST. We obtain additional insight into the structures as comprising $A B A B$ squares (and cubes) [Fig. 8(b)], and the relationship to the perfectly ordered $A B A B$ squares of the rocksalt phase is clear. Closer inspection of the local environment of the cubic subunit in the center of the cell reveals that there are intermediate distances where a slight reorientation would result in even more $A B$ bonds and $A B A B$ squares, corresponding to the above mentioned bridges of the distance-distance correlation map (Fig. 3). Integration of the corresponding radial correlation function up to the shifted first minimum indicates that there are 1.3 (2.0) intermediate distances per $\mathrm{Ge}(\mathrm{Sb})$ atom in $a$-GST, and the value is $1.0(\mathrm{Ge})$ for $a$-GeTe.

\section{Vacancies}

We have noted that vacancies play important roles in phase-change materials. The analysis of cavities or "voids" reveals a wide range of volumes and shapes, with a total volume of $11.8 \%$ and $6.4 \%$ for $a$-GST and $a$-GeTe, respectively. GeTe then contains numerous cavities (vacancies) in the amorphous phase, whereas the ideal crystalline phase has 

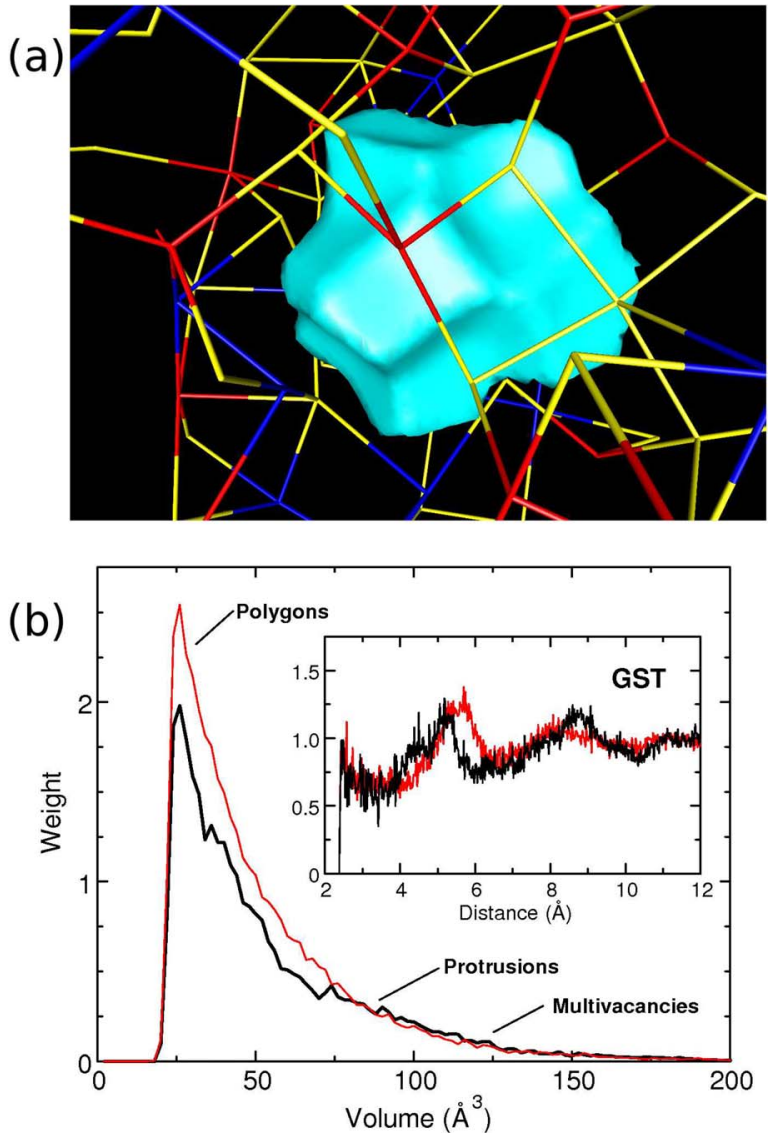

FIG. 9. (Color online) (a) A medium-sized cavity. (b) Volume distribution of cavities in $a$-GST, and (inset) vacancy-vacancy correlation function. Red, liquid at $900 \mathrm{~K}$; black, $a$-GST at $300 \mathrm{~K}$.

none. The vacancies are surrounded mainly by Te atoms, as in the crystalline phases of pseudobinary GeSbTe compounds, and a medium-sized cavity in $a$-GST is shown in Fig. 9(a). The sharp corners and edges are typical, and the protrusions are caused by oriented vacancy domains that reflect the semiordered structure of the material.

The volume distribution of cavities in $a$-GST and the vacancy-vacancy correlation function (inset) are plotted in Fig. 9(b). The broad distribution is biased toward small cavities, the shapes of which resemble polygons or Voronoi polyhedra (Sec. II B) due to the small vacancy domains. At larger volumes $\left(50-100 \AA^{3}\right)$, these domains often involve protrusions, and di- and multivacancies become important. Insertion of "test spheres" into the cavities shows that their interaction is repulsive (there are few divacancies). There are long-range vacancy-vacancy correlations to at least $10 \AA$ [Fig. 9(b), inset] that are similar to the behavior in Te, the preferred neighbor of vacancies. Comparison of amorphous $(300 \mathrm{~K})$ and liquid GST $(900 \mathrm{~K})$ shows that the latter contains more small cavities (total volume of 13.8\%), but the distributions of large cavities are almost identical. Correlations are still evident at $900 \mathrm{~K}$ (to $\sim 7 \AA$ ), and the dominant peak is shifted from $5.1 \AA(300 \mathrm{~K})$ to $5.7 \AA(900 \mathrm{~K})$.

The rapid amorphous-to-crystalline transition can be viewed as a vacancy-supported reorientation of $A B A B$ squares. Numerous $A B$ bonds are formed, but few homopolar

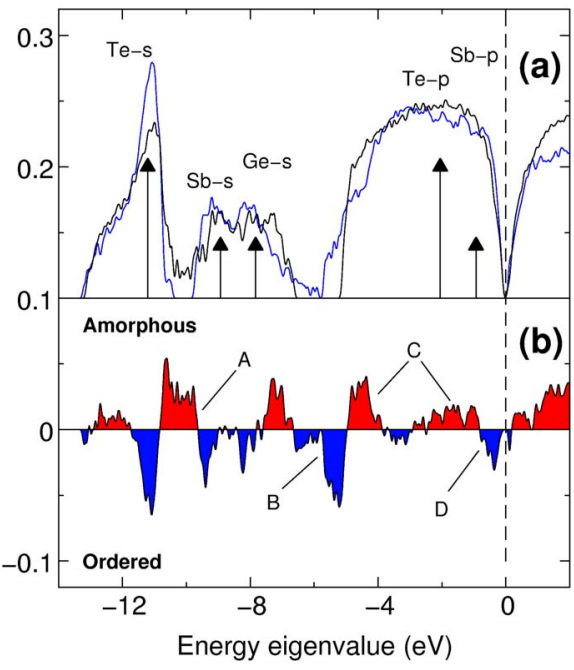

FIG. 10. (Color online) (a) Electronic DOS. Black, $a$-GST; blue, $c$-GST. Atomic eigenvalues (arrows) are shifted uniformly so that the Ge $p$ eigenvalue corresponds to Fermi energy (vertical dashed line). (b) DOS difference. Red, $a$-GST dominates; blue, $c$-GST dominates. See text for labels A, B, C, and D.

bonds need to be broken in order to achieve crystalline order. This picture is consistent with the pressure-induced amorphization experiments of Kolobov et al. ${ }^{36}$ which show that the phase transition in GST involves relatively small atomic displacements and a strong second-nearest neighbor interaction between Te atoms. By contrast, the absence of vacancies in $c$-GeTe means that it does not amorphize on compression.

\section{E. Electronic structure}

Optimizing the geometries of the amorphous phases has enabled us to perform the first calculations of their electronic structures. The electronic DOS of $a$ - and $c$-GST and their difference are shown in Fig. 10. The band gaps at the Fermi energy ( $a$-GST: $0.19 \mathrm{eV}$ and $c$-GST: $0.18 \mathrm{eV}$ ) are almost identical and less than the measured optical band gaps in the amorphous $(0.7 \mathrm{ev}), \mathrm{fcc}(0.5 \mathrm{eV})$, and hexagonal $(0.5 \mathrm{eV})$ phases. ${ }^{37}$ This is a common situation in DF calculations, and spin-orbit corrections further reduce the gap in $c$-GeTe. ${ }^{38}$ Amorphization in semiconductors usually results in broader valence and conduction bands that can overlap, so a larger band gap is unusual. ${ }^{10}$

The overall form of the DOS (Fig. 10) can be understood in terms of a simple model for systems with average valence of $5(\mathrm{GeTe})$ or near this value (GST). If the $p$ electrons assigned to $\mathrm{Ge}$ (and $\mathrm{Sb}$ in GST) are transferred to Te, they will fill each atomic $5 p$ shell. In a rocksalt geometry, a $p^{3}$-type interaction between neighboring atoms results in a set of $3 N(\mathrm{Te})$ doubly occupied bonding $\pi$ orbitals, where $N(\mathrm{Te})$ is the number of Te atoms. The calculated DOS of $c$-GST shows indeed $\sigma$ bands derived from atomic $s$ components and a broad $\pi$ band from $-6 \mathrm{eV}$ to the Fermi energy. For the $\mathrm{NaCl}$ structure, $s$ and $p$ states interact only at $\mathbf{k}$ points that are not of high symmetry, ${ }^{38}$ and the overlap between the $\sigma$ and $\pi$ bands is small. The projections onto atomic orbitals 


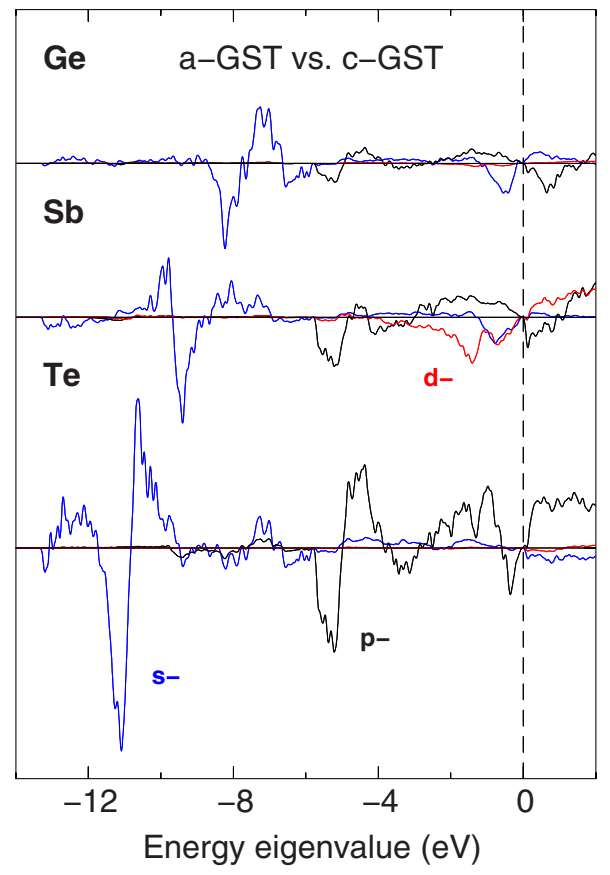

FIG. 11. (Color online) Projected DOS difference (atomic orbitals) in $a$-GST and $c$-GST. Blue, $s$; black, $p$; and red, $d$ components. Positive values refer to a larger weight in $a$-GST.

then change suddenly from $s$ to $p$ character. The number of $p$ electrons equals $6 N(\mathrm{Te})$ for all GeSbTe stoichiometries along the pseudobinary line $\mathrm{GeTe}-\mathrm{Sb}_{2} \mathrm{Te}_{3}$, resulting in a band gap at the Fermi energy for crystal structures based on the rocksalt motif (fcc).${ }^{14}$ For these alloys, the presence of vacancies breaks the periodic $p^{3}$-bonding pattern of the fcc phase, yielding bands of antibonding character below the Fermi energy (from -1 to $0 \mathrm{eV}$ ). ${ }^{14}$ Visualization of the orbitals involved confirmed that this is also true in the present work.

The DOS difference plot [Fig. 10(b)] has four important regions (A, B, C, and D). The first (A) shows a merging of the lower (Te, "bonding") and intermediate ( $\mathrm{Sb} / \mathrm{Ge}$, "antibonding") $\sigma$ bands in $a$-GST following the shortening of bonds that increases the $s-s$ interaction between $\mathrm{Sb}$ and $\mathrm{Te}$. Another change (B) is observed near $-6 \mathrm{eV}$, where $a$-GST has a large band gap between the intermediate $\sigma$ band and the $\pi$ band, followed by an increased weight in the $\pi$ band for $a$-GST (C), as partial $s p^{3}$ hybridization of Ge atoms occurs and the number of neighbors decreases. Analysis of the atomic projections indicates that the main component associated with this change is Te $p$. The final area (D) at the Fermi energy has a lower weight for the amorphous structure consistent with the measured change in optical properties. Figure 11 shows that $\mathrm{Ge} s, \mathrm{Sb} s, \mathrm{Sb} p, \mathrm{Sb} d$, and $\mathrm{Te} p$ components are important, and the larger weight of the conduction band in $a$-GST can be traced to $\mathrm{Sb}$ and Te atoms. $\mathrm{Sb} d$ contributes to both the valence and conduction $\pi$ bands, whereas the Ge $d$ and Te $d$ components are negligible.

Although most Ge atoms are not "tetrahedral," the overall differences between the amorphous and ordered phases can be understood in terms of a change from $s p^{3}$ bonding (low coordination) to $p^{3}$ bonding (high coordination). This results in a narrower $\pi$ band for $a$-GST and a reduced weight at the

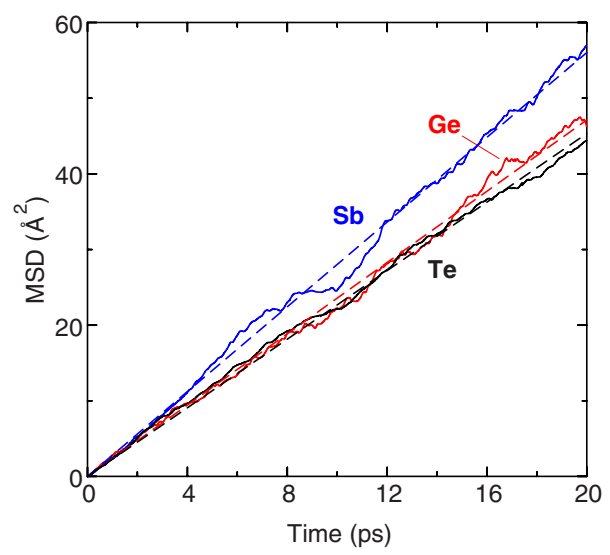

FIG. 12. (Color online) The mean square displacement of different atomic types of GST at $900 \mathrm{~K}$ (melting point). The dashed lines are linear fits for each element.

Fermi energy as the band gap opens. The projected DOS of tetrahedrally (amorphous) and octahedrally (crystal) coordinated $\mathrm{Ge}$ atoms supports this interpretation.

\section{F. Diffusion}

The mean square displacements of different elements at $900 \mathrm{~K}$ and the corresponding linear fits are shown in Fig. 12. The modulations of linear behavior between 5 and 12 ps (see $\mathrm{Sb}$ ) correlate with the number of cavities (total volume), indicating concerted changes in the material as cavities form, merge, and annihilate. The diffusion coefficients $D$ [Eq. (11)] are $3.93 \times 10^{-5}(\mathrm{Ge}), \quad 4.67 \times 10^{-5}(\mathrm{Sb}), \quad$ and 3.78 $\times 10^{-5} \mathrm{~cm}^{2} \mathrm{~s}^{-1}(\mathrm{Te})$. The coordination number of $\mathrm{Sb}$ in the melt (3.4) is lower than in $a$-GST, and it is the most mobile element.

The diffusion coefficients of GeTe at $1000 \mathrm{~K}$ are 4.65 $\times 10^{-5}(\mathrm{Ge})$ and $3.93 \times 10^{-5} \mathrm{~cm}^{2} \mathrm{~s}^{-1}(\mathrm{Te})$. Earlier DF simulations for liquid GeTe reported diffusion constants that are significantly lower $\left(1.5 \times 10^{-5}\right.$ and $0.4 \times 10^{-5}$ for Ge and $\mathrm{Te}$, respectively). ${ }^{39}$ This difference from our result may arise from the shorter (1.6 ps) and smaller (64 atoms in the cell) simulation. Our calculated viscosity ranges between 1.1 and $1.2 \mathrm{cP}$ for both alloys, depending on the particle radius chosen in the Stokes-Einstein relation. The measured viscosity of liquid GeTe ranges from $\sim 1.9 \mathrm{cP}$ at $1000 \mathrm{~K}$ to $1.3 \mathrm{cP}$ at $1150 \mathrm{~K}^{40}$

\section{CONCLUDING REMARKS}

Density functional calculations combined with molecular dynamics have been performed for liquid and amorphous forms of $\mathrm{GeTe}$ and $\mathrm{Ge}_{2} \mathrm{Sb}_{2} \mathrm{Te}_{5}$. The simulations involve 216 and 460 atoms, respectively, in the unit cell, over hundreds of picoseconds, i.e., a time scale similar to the quenching process from the liquid. Amorphous GeTe and GST show long-range ordering of Te atoms and a high degree of alternating four-membered rings ( $A B A B$ squares) that are the main building blocks of both. Since the crystalline (rocksalt) phase comprises perfectly ordered $A B A B$ squares, we can 
view the rapid amorphous-to-crystalline transition as a reorientation of $A B A B$ squares to form additional $A B$ bonds and cubic subunits in a locally "distorted octahedral" structure. Vacancies with characteristically sharp edges, corners, and protrusions play a crucial role in providing the necessary space. If we include intermediate $A B$ distances in determining the coordination numbers in $a$-GST, they increase to 5.5 $(\mathrm{Ge}), 5.7(\mathrm{Sb})$, and $4.2(\mathrm{Te})$, which are close to the crystalline values of 6,6 , and 4.8 , respectively.

$\mathrm{Ge}_{2} \mathrm{Sb}_{2} \mathrm{Te}_{5}$ is used in DVD-RAM, and there are several reasons why its phase-change properties are superior to those of the prototype phase-change material GeTe:

(1) Vacancies. The rhombohedral and fcc forms of GeTe are vacancy-free, and $a$-GeTe contains $6.4 \%$ vacancies by volume, about half of the value for $a$-GST. Recently, Wuttig et al. ${ }^{14}$ demonstrated for several GST stoichiometries that the energetically optimal concentration of vacancies is above $10 \%$ for the ordered rocksalt structures (as in GST). The local similarity between the amorphous and ordered phases is greater in GST than in GeTe due to the higher concentration of vacancies.

(2) Homopolar bonds and $A B$ ordering. There are more homopolar Ge-Ge bonds in $a$-GeTe (1.1) than $A-A$ bonds (Ge-Ge, Ge-Sb, and Sb-Sb) in $a$-GST ( 0.6 for Ge and 0.8 for $\mathrm{Sb})$. This leads to different $A B$ ordering and ring statistics, and $A B A B$ squares are more evident in $a$-GST. Furthermore, the change in the local coordination of Ge atoms means that the tetrahedral $\mathrm{Ge}_{-} \mathrm{Te}_{4}$ units occur more often in $a$-GST. The differences arise from the lower concentration of vacancies in $a$-GeTe, which increases the Ge-Ge $(A-A)$ interactions.

(3) Interatomic distances. The first minima of the radial $\mathrm{Ge}-\mathrm{Te}$ and $\mathrm{Sb}$-Te distribution functions of $a$-GST extend to 3.8-3.9 $\AA$, and these intermediate distances make possible new bonds of type $A B$. This feature is less pronounced in $a$-GeTe, where there are fewer vacancies and the minimum is at $3.6 \AA$.
(4) Atomic mobility. The molten phases of $a$-GST (at $900 \mathrm{~K}$ ) and $a$-GeTe (at $1000 \mathrm{~K}$ ) have different atomic mobilities. This can be traced to the $\mathrm{Sb}$ atoms, whose diffusion constants are $\sim 20 \%$ larger than those of Ge and Te (see Fig. 12).

(5) Electronic structure. The presence of Sb in GST adds significant $d$ components to the valence and conduction $\pi$ bands. Shortened bonds in the amorphous phase also increase the $s-s$ interaction between $\mathrm{Sb}$ and Te in $a$-GST, and the weight of $\mathrm{Sb}$ in the conduction band affects the optical properties. Deviations from the pseudobinary line enhance the optical contrast, as the $6 N(\mathrm{Te})$ rule of the rocksalt phase is not satisfied, and there is a finite valence electron density at the Fermi energy. ${ }^{14}$

The development of phase-change memory devices has favored chalcogenide semiconductors from the beginning. It is striking, however, that the current focus of research and development is almost entirely on Te-based alloys. To understand the special role of this element, we are carrying out simulations on both the eutectic alloy $\mathrm{Ge}_{15} \mathrm{Te}_{85}$ and Te itself in the amorphous and liquid states. Preliminary results are consistent with the picture given above, and the coordination of Te is close to threefold in all cases.

\section{ACKNOWLEDGMENTS}

J.A. acknowledges financial support from European Union project ULTRA-1D (NMP4-CT-2003-505457). The calculations were performed on IBM Blue Gene/L and p690 computers in the Forschungszentrum Jülich with grants from the FZ Jülich and the John von Neumann Institute for Computing (NIC). We thank M. Wuttig and W. Wełnic for helpful discussions, and I. Kaban, T. Matsunaga, M. Parrinello, and Y. Tsuchiya for providing information about published and unpublished work.
${ }^{1}$ A. L. Greer and N. Mathur, Nature (London) 437, 1246 (2005).

${ }^{2}$ M. Wuttig, Nat. Mater. 4, 265 (2005).

${ }^{3}$ M. H. R. Lankhorst, B. W. S. M. M. Ketelaars, and R. A. M. Wolters, Nat. Mater. 4, 347 (2005).

${ }^{4}$ H. F. Hamann, M. O’Boyle, Y. C. Martin, M. Rooks, and H. K. Wickramasinghe, Nat. Mater. 5, 383 (2006).

${ }^{5}$ S. R. Ovshinsky, Phys. Rev. Lett. 21, 1450 (1968).

${ }^{6}$ M. Chen, K. A. Rubin, and R. W. Barton, Appl. Phys. Lett. 49, 502 (1986).

${ }^{7}$ J. K. Olson, H. Li, and P. C. Taylor, J. Ovonic Res. 1, 1 (2005).

${ }^{8}$ N. Yamada, MRS Bull. 21, 48 (1996).

${ }^{9}$ A. Kolobov, P. Fons, A. I. Frenkel, A. I. Ankudinov, J. Tomonaga, and T. Uruga, Nat. Mater. 3, 703 (2004), and references therein.

${ }^{10}$ W. Wełnic, A. Pamungkas, R. Detemple, C. Steimer, S. Blügel, and M. Wuttig, Nat. Mater. 5, 56 (2006).

${ }^{11}$ S. Hosokawa, T. Ozaki, K. Hayashi, N. Happo, M. Fujiwara, K. Horii, P. Fons, A. V. Kolobov, and J. Tominaga, Appl. Phys. Lett. 90, 131913 (2007).

${ }^{12}$ D. A. Baker, M. A. Paesler, G. Lucovsky, S. C. Agarwal, and P.
C. Taylor, Phys. Rev. Lett. 96, 255501 (2006).

${ }^{13}$ T. Matsunaga, R. Kojima, N. Yamada, K. Kifune, Y. Kubota, Y. Tabata, and M. Takata, Inorg. Chem. 45, 2235 (2006).

${ }^{14}$ M. Wuttig, D. Lüsebrink, D. Wamwangi, W. Wełnic, M. Gilleßen, and R. Dronskowski, Nat. Mater. 6, 122 (2007).

${ }^{15}$ Z. Sun, J. Zhou, and R. Ahuja, Phys. Rev. Lett. 98, 055505 (2007).

${ }^{16}$ C. Bichara, M. Johnson, and J. P. Gaspard, Phys. Rev. B 75, 060201(R) (2007).

${ }^{17}$ S. Kohara, K. Kato, S. Kimura, H. Tanaka, T. Usuki, K. Suzuya, H. Tanaka, Y. Moritomo, T. Matsunaga, N. Yamada, Y. Tanaka, H. Suematsu, and M. Takata, Appl. Phys. Lett. 89, 201910 (2006).

${ }^{18}$ K. Kohary, V. M. Burlakov, and D. G. Pettifor, Phys. Rev. B 71, 235309 (2005). Bond switching algorithms have also been used to describe, for example, amorphization in $\mathrm{Si}$ and $\mathrm{Ge}[\mathrm{F}$. Wooten, K. Winer, and D. Weaire, Phys. Rev. Lett. 54, 1392 (1985)] and ring-chain equilibria in polymers [P. Ballone and R. O. Jones, J. Chem. Phys. 115, 3895 (2001)].

${ }^{19}$ See, for example, D. Hohl and R. O. Jones, Phys. Rev. B 43, 
3856 (1991); X. Zhang and D. A. Drabold, Phys. Rev. Lett. 83, 5042 (1999) [both amorphous Se]; R. O. Jones and P. Ballone, J. Chem. Phys. 118, 9257 (2002) [S $\mathrm{S}_{n}$ clusters to $\left.n=18\right]$; L. Giacomazzi, C. Massobrio, and A. Pasquarello, Phys. Rev. B 75, 174207 (2007) [ $\left.\mathrm{GeSe}_{2}\right]$.

${ }^{20}$ CPMD, Version 3.11, IBM Corp., 1990-2006, MPI für Festkörperforschung Stuttgart, 1997-2001 (http://www.cpmd.org).

${ }^{21}$ J. P. Perdew, K. Burke, and M. Ernzerhof, Phys. Rev. Lett. 77, 3865 (1996).

${ }^{22}$ N. Troullier and J. L. Martins, Phys. Rev. B 43, 1993 (1991).

${ }^{23}$ S. G. Louie, S. Froyen, and M. L. Cohen, Phys. Rev. B 26, 1738 (1982).

${ }^{24}$ J. Hutter, H.-P. Lüthi, and M. Parrinello, Comput. Mater. Sci. 2, 244 (1994).

${ }^{25}$ A. I. Lichtenstein, R. O. Jones, S. de Gironcoli, and S. Baroni, Phys. Rev. B 62, 11487 (2000).

${ }^{26}$ A. Alavi, J. Kohanoff, M. Parrinello, and D. Frenkel, Phys. Rev. Lett. 73, 2599 (1994).

${ }^{27}$ Low Energy Neutrons and their Interaction with Nuclei and Matter, edited by H. Schopper, Landolt-Börnstein, New Series, Group I, Vol. 16, Pt. A (Springer, Berlin, 2000), Chap. 6.

${ }^{28}$ H. Ruppersberg and C. N. J. Wagner, J. Non-Cryst. Solids 55, 165 (1981), and references therein.

${ }^{29}$ See, for example, R. Yamamoto and A. Onuki, Phys. Rev. Lett. 81, 4915 (1998).
${ }^{30}$ Y. Maeda and M. Wakagi, Jpn. J. Appl. Phys., Part 1 30, 101 (1991).

${ }^{31}$ T. Chattopadhyay, J. X. Boucherle, and H. G. von Schnering, J. Phys. C 20, 1431 (1987).

${ }^{32}$ M. Delheusy, J. Y. Raty, R. Detemple, W. Wełnic, M. Wuttig, and J.-P. Gaspard, Physica B 350, 1055(E) (2004).

${ }^{33}$ Fourfold coordinated Ge atoms are "tetrahedral" if all six Gecentered bond angles are $>100^{\circ}$.

${ }^{34}$ N. Rivier, Philos. Mag. A 40, 859 (1979).

${ }^{35}$ F. Wooten, Acta Crystallogr., Sect. A: Found. Crystallogr. 58, 346 (2002).

${ }^{36}$ A. V. Kolobov, J. Haines, A. Pradel, M. Ribes, P. Fons, J. Tominaga, Y. Katayama, T. Hammouda, and T. Uruga, Phys. Rev. Lett. 97, 035701 (2006).

${ }^{37}$ B.-S. Lee, J. R. Abelson, S. G. Bishop, D.-H. Kang, B-K. Cheong, and K.-B. Kim, J. Appl. Phys. 97, 093509 (2005).

${ }^{38}$ A. H. Edwards, A. C. Pineda, P. A. Schultz, M. G. Martin, A. P. Thompson, H. P. Hjalmarson, and C. J. Umrigar, Phys. Rev. B 73, 045210 (2006).

${ }^{39}$ J. Y. Raty, V. V. Godlevsky, J. P. Gaspard, C. Bichara, M. Bionducci, R. Bellissent, R. Céolin, J. R. Chelikowsky, and P. Ghosez, Phys. Rev. B 65, 115205 (2002).

${ }^{40}$ V. M. Glazov, S. N. Chizhevskaya, and N. N. Glagoleva, Liquid Semiconductors (Plenum, New York, 1969), p. 205. 